\title{
A Flexible and Ultra-Highly Sensitive Tactile Sensor through Parallel Circuit by a Magnetic Aligned Conductive Composite
}

Yang Jiang ${ }^{1,2 \ddagger}$, Fei Liang ${ }^{1,2 \ddagger}$, Hua Yang $\mathrm{Li}^{3,4 *}$, Xin $\mathrm{Li}^{1,2}$, You Jun $\mathrm{Fan}^{1,2}$, Jin Wei Cao ${ }^{3,4}$, Yi Ming Yin ${ }^{3}$, Ying Wang ${ }^{1,2}$, Zhong Lin Wang ${ }^{1,2,5,6 *}$, and Guang Zhu ${ }^{1,2,3 *}$

1. CAS Center for Excellence in Nanoscience, Beijing Key Laboratory of Micro-Nano Energy and Sensor, Beijing Institute of Nanoenergy and Nanosystems, Chinese Academy of Sciences, Beijing 100083, P. R. China.

2. School of Nanoscience and Technology, University of Chinese Academy of Sciences, Beijing 100049, P. R. China.

3. New Materials Institute, Department of Mechanical, Materials and Manufacturing Engineering, University of Nottingham Ningbo China, Ningbo 315100, P. R. China.

4. CAS Key Laboratory of Magnetic Materials and Devices, Ningbo Institute of Materials Technology and Engineering Chinese Academy of Sciences, Ningbo, 315201, China.

5. CUSTech Institute, Wenzhou, Zhejiang, 325024, China.

6. School of Materials Science and Engineering, Georgia Institute of Technology, Atlanta, GA 30332-0245, USA.

‡ These authors contributed equally to this work.

*Corresponding author: Huayang Li (Huayang.Li@nottingham.edu.cn), Zhong Lin Wang (zlwang@gatech.edu), and Guang Zhu (Guang.Zhu@nottingham.edu.cn). 


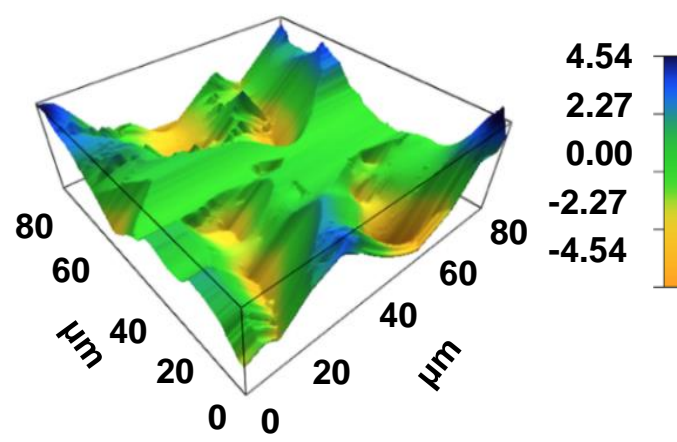

Figure S1. AFM of the surface of nickel-coated carbon fibers (NICFs)/polydimethylsiloxane (PDMS) electrodes.

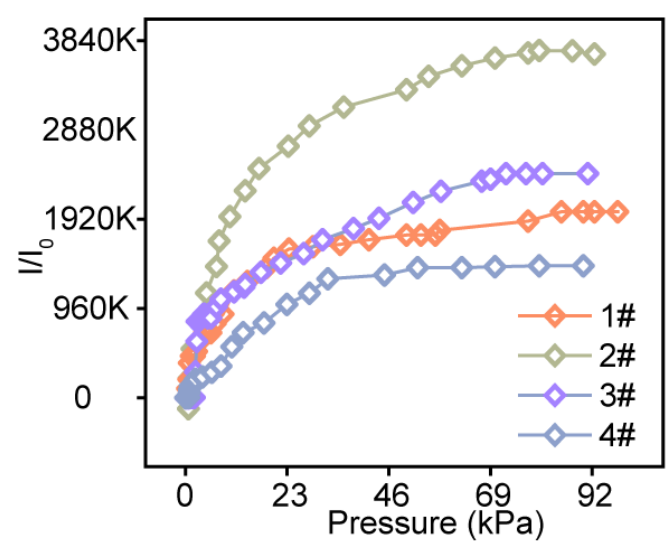

Figure S2. Sensitivity characterization of sensors with different length of NICFs. (1\# $0.05 \mathrm{~mm}, 2 \# 0.1 \mathrm{~mm}, 3 \# 0.2 \mathrm{~mm}, 4 \# 0.3 \mathrm{~mm})$.
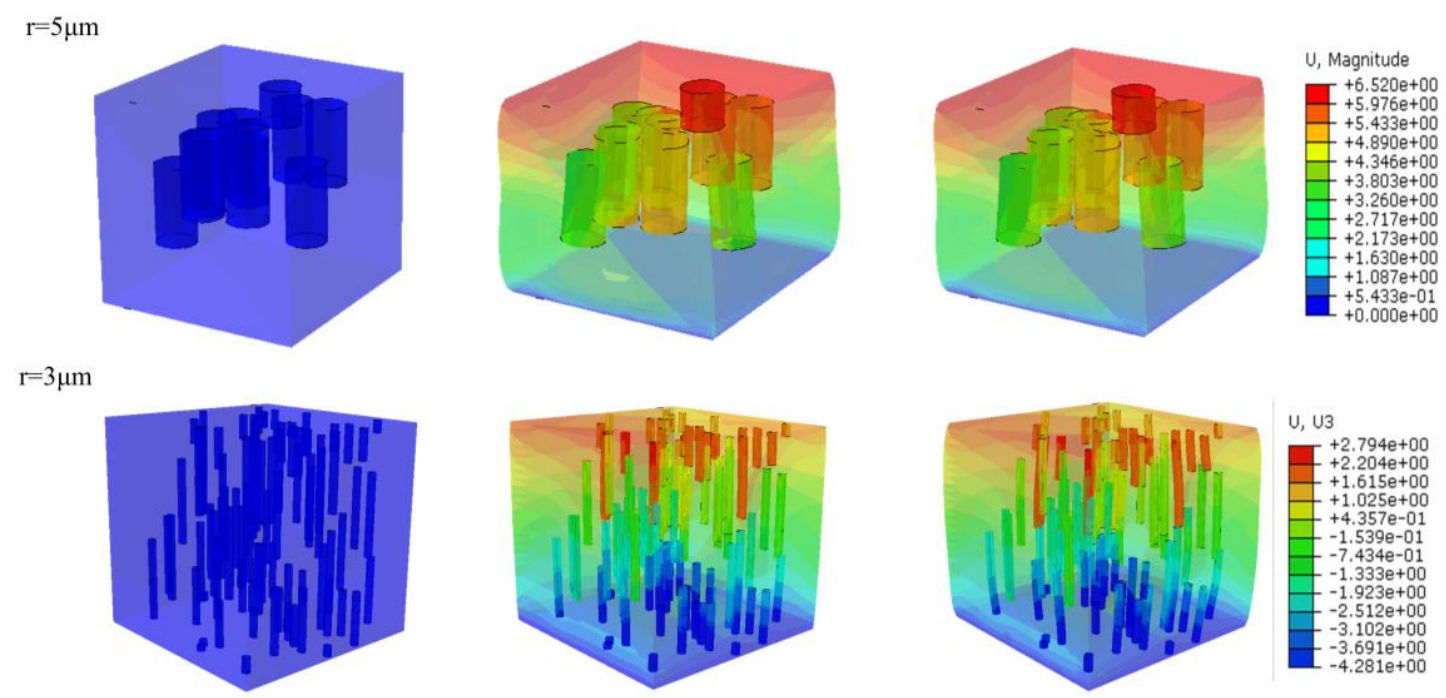

Figure S3. The deformation distribution of NICFs ( $3 \mu \mathrm{m}, 5 \mu \mathrm{m}$ in diameter) with the 
applied displacement through representative volume element (RVE) analysis.

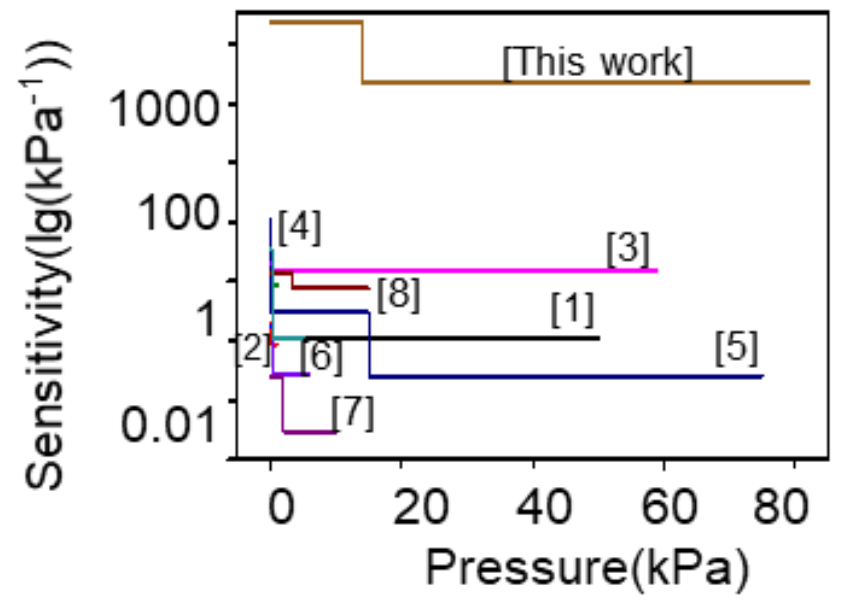

Figure S4. Comparison of the sensitivities within a linear pressure range between the oriented NICFs/PDMS pressure sensor and previous reported surface patterned microstructures. ${ }^{1-8}$

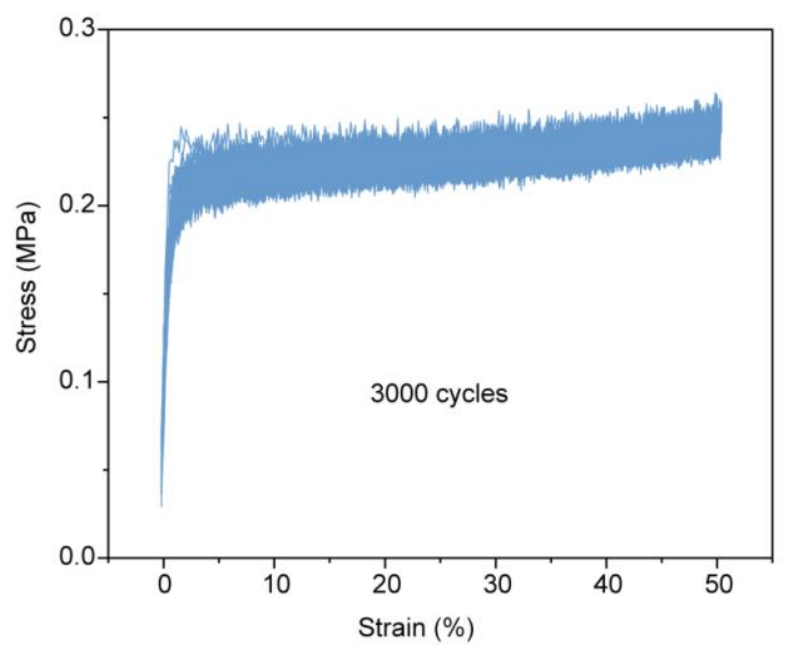

Figure S5. Cyclic stress-strain curve of the alignment NICFs composite.
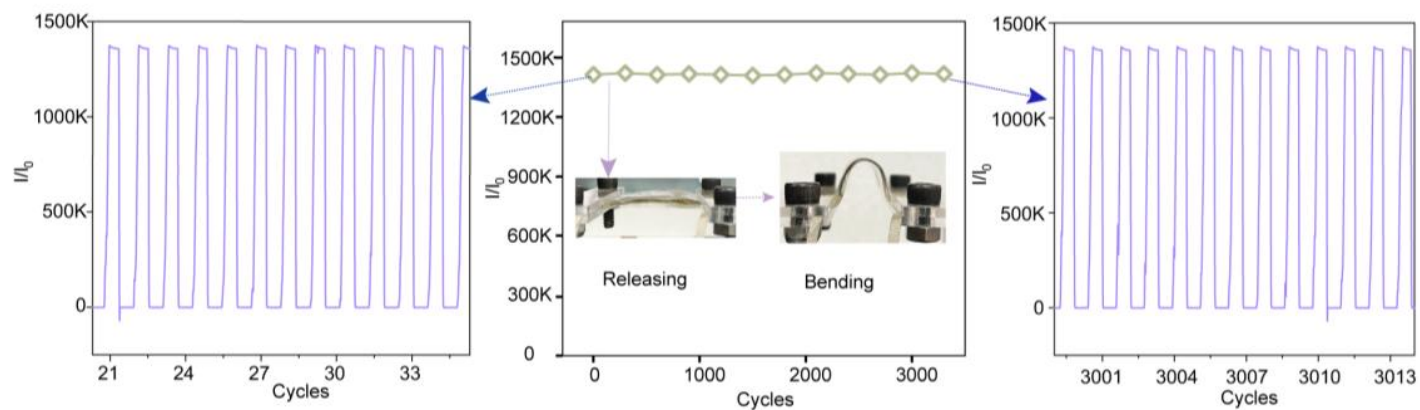

Figure S6. Bending cycles of the MAPS. 

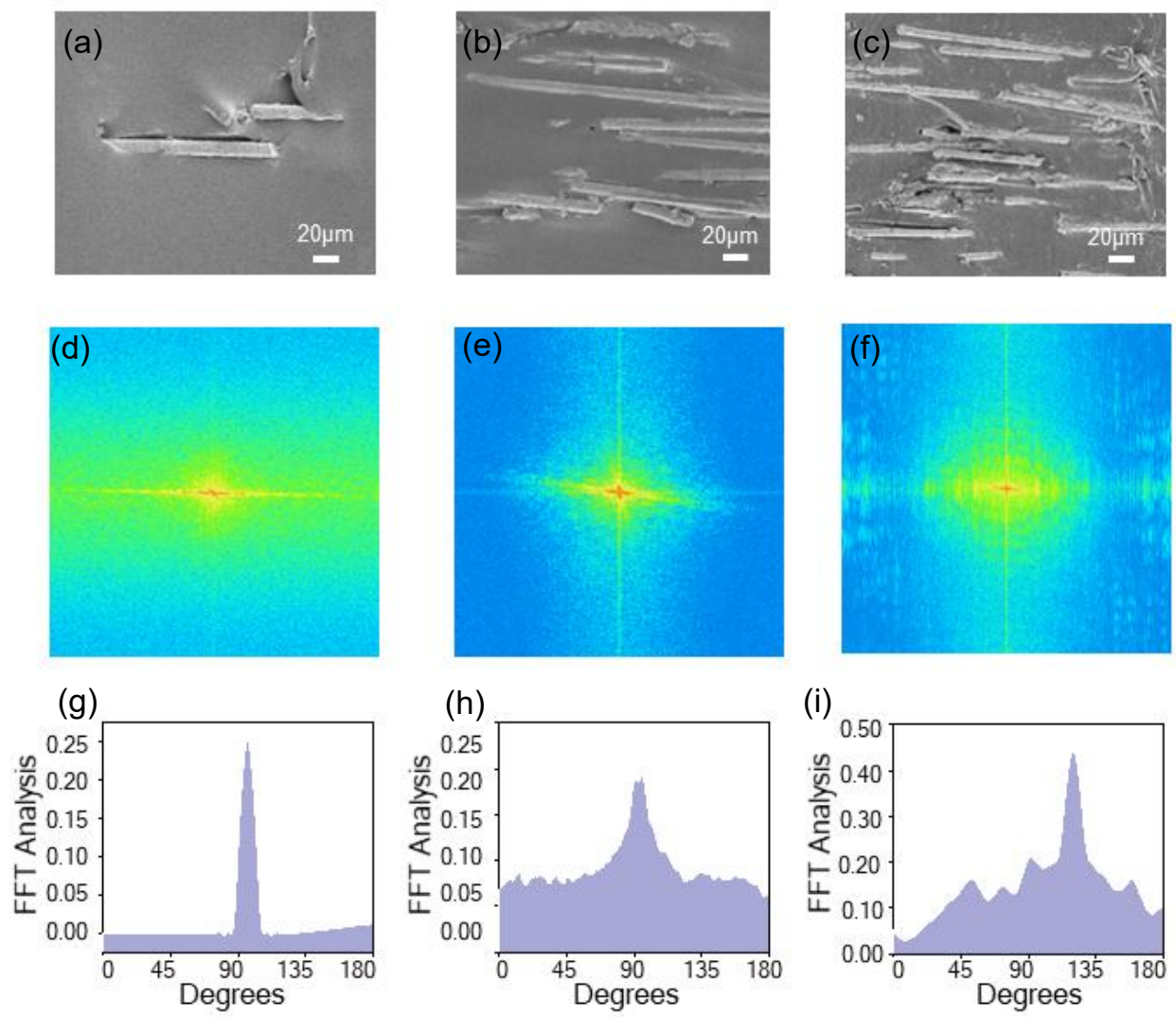

Figure S7. SEM and FFT analysis of different concentration NICFs/PDMS composite.

(a)Scanning Electron Microscope (SEM) of Aligned NICFs/PDMS composite (NICF 11\%). (b) Scanning Electron Microscope (SEM) of Aligned NICFs/PDMS composite (NICF 16\%). (c) Scanning Electron Microscope (SEM) of Aligned NICFs/PDMS composite (NICF 20\%). (d-f) 2D FFT analysis of the degree of NICFs. (g-i) 2D FFT alignment plots for the corresponding Scanning Electron Microscope.

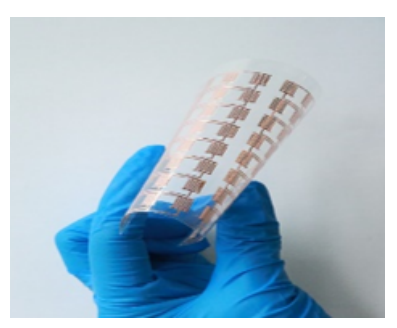

Figure S8. The photograph of the interdigitated electrode. 
a

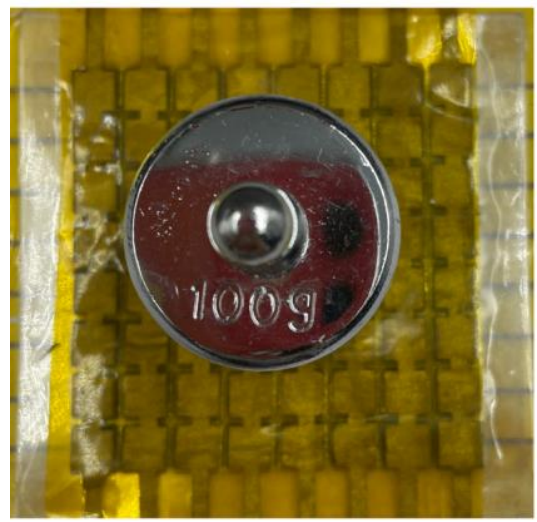

C

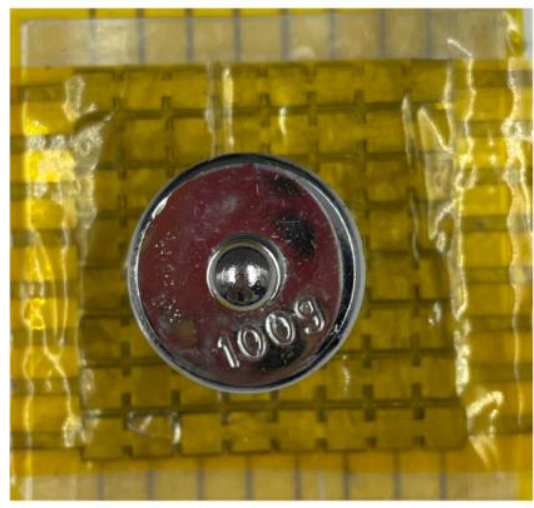

b

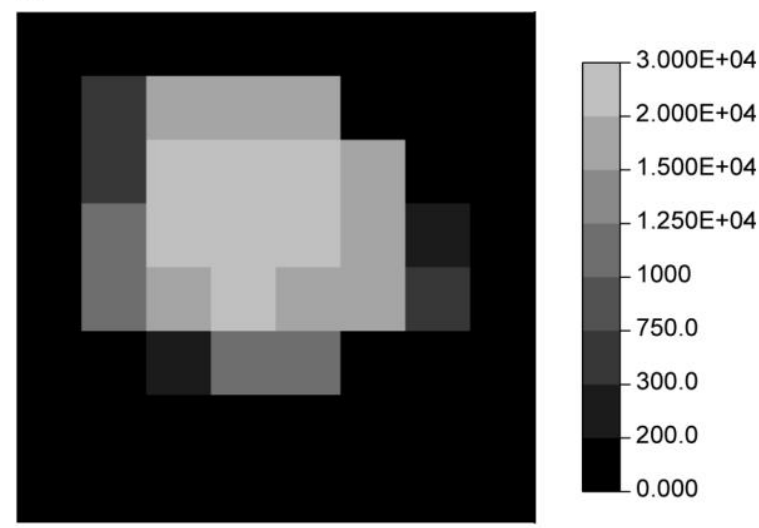

d

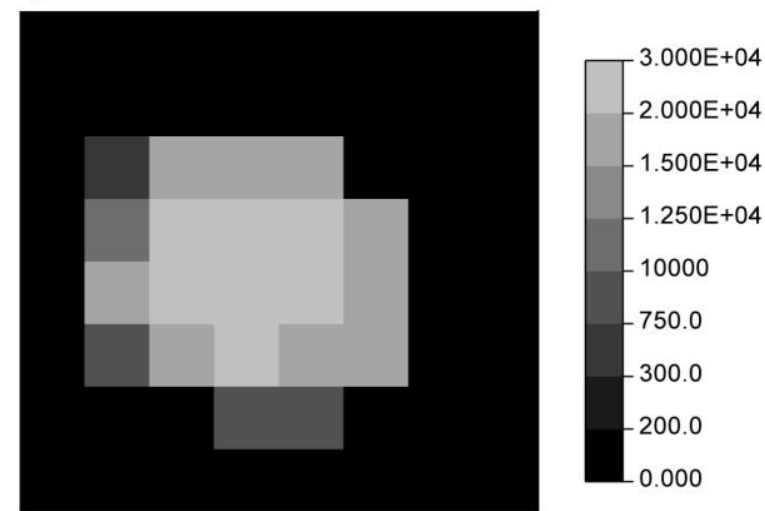

Figure S9. Different devices of $8 \times 8$ sensing arrays. 
(a)

(c)
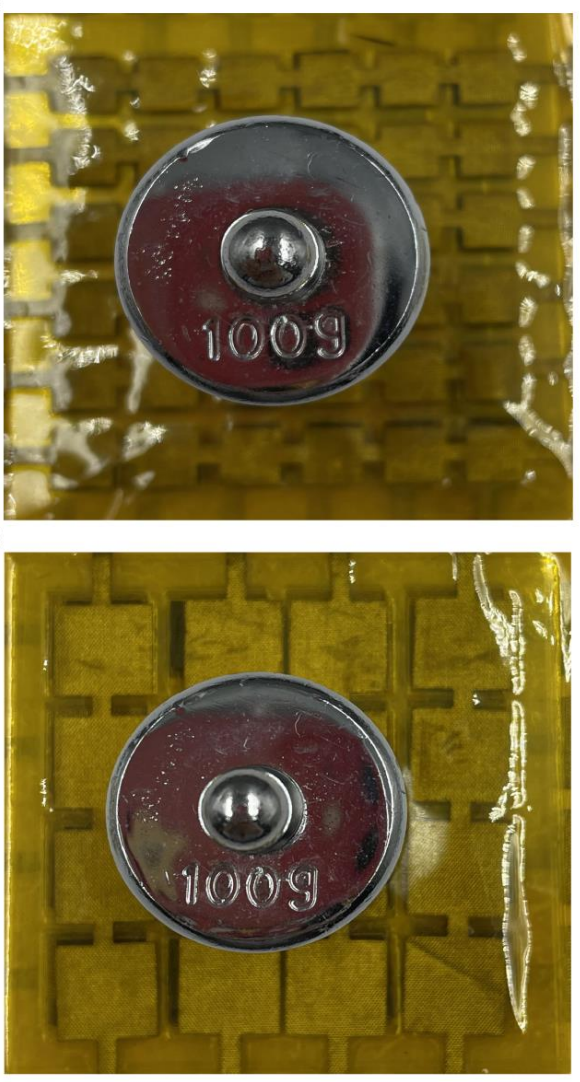

b)

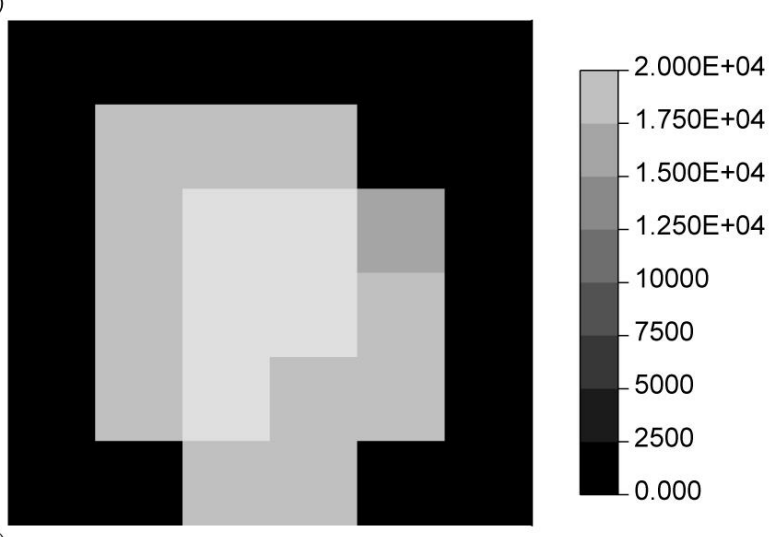

d)

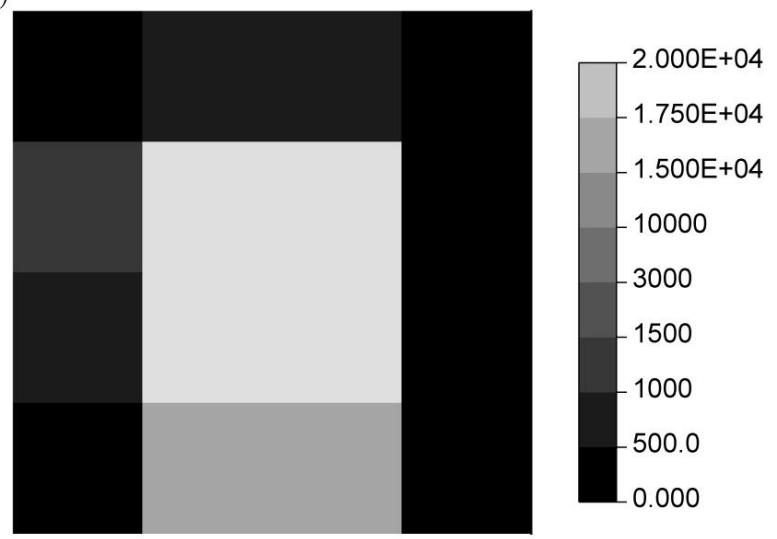

Figure S10. (a-b) $6 \times 6$ sensing arrays. (c-d) $4 \times 4$ sensing arrays.
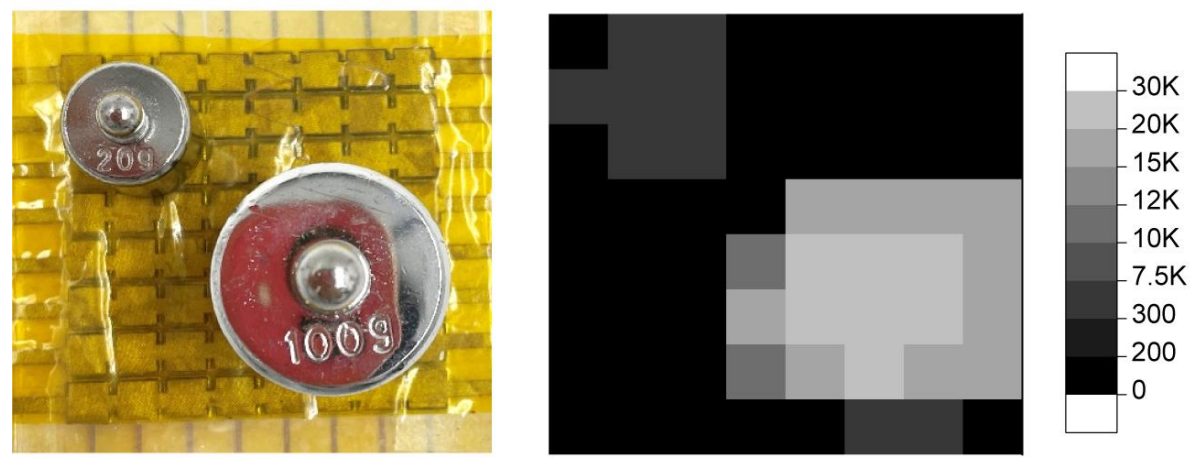

Figure S11. Schematics of the detection of applied pressure from different weights of loads and their corresponding. 


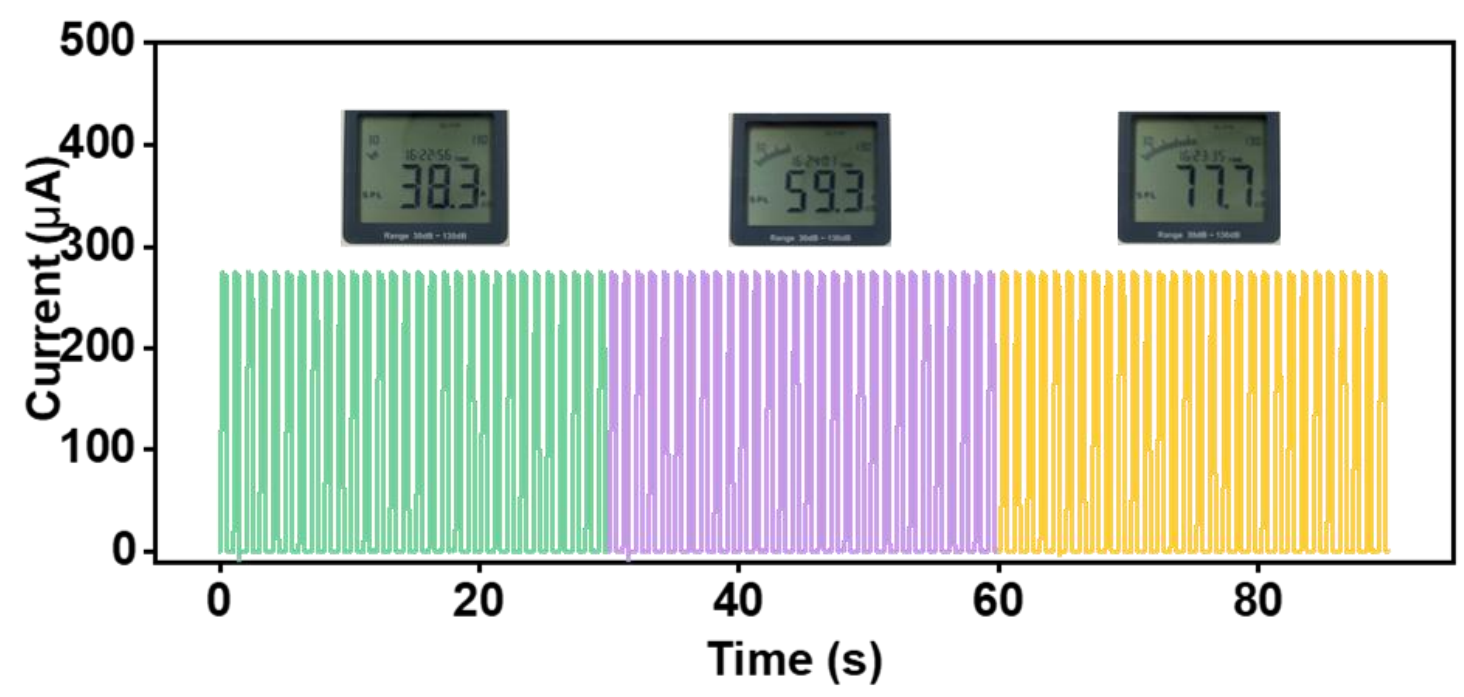

Figure S11. The electronic response to different noise.

\begin{tabular}{|c|c|c|c|c|}
\hline Sensing Materials & Sensitivity & Detection Range & Response Time & Reference \\
\hline PTNWs/Graphene & $9.4 \times 10^{-3} \mathrm{kPa}^{-1}$ & $0-1.5 \mathrm{kPa}$ & $5-7 \mathrm{~ms}$ & 9 \\
\hline rGO/PVDF & $15.6 \mathrm{kPa}^{-1}$ & $1.2 \mathrm{~Pa}-60 \mathrm{kPa}$ & $5 \mathrm{~ms}$ & 10 \\
\hline rGO & $0.8 \mathrm{kPa}^{-1}$ & $0.24 \mathrm{~Pa}-4 \mathrm{kPa}$ & $<100 \mathrm{~ms}$ & 11 \\
\hline LSG & $0.96 \mathrm{kPa}^{-1}$ & $10-100 \mathrm{kPa}$ & $0.4 \mathrm{~ms}$ & 12 \\
\hline ACNT/Graphene & $19.8 \mathrm{kPa}^{-1}$ & $0.6 \mathrm{~Pa}-0.3 \mathrm{kPa}$ & $<16.7 \mathrm{~ms}$ & 13 \\
\hline WG & $6.92 \mathrm{kPa}^{-1}$ & $0-5 \mathrm{kPa}$ & - & 14 \\
\hline MX/rGO & $22.56 \mathrm{kPa}^{-1}$ & $0-3.5 \mathrm{kPa}$ & $<200 \mathrm{~ms}$ & 15 \\
\hline rGO/PU & $0.26 \mathrm{kPa}^{-1}$ & $0-10 \mathrm{kPa}$ & - & 7 \\
\hline rGO/PI/HT & $0.36 \mathrm{kPa}^{-1}$ & $0.2 \mathrm{~Pa}-14 \mathrm{kPa}$ & $<80 \mathrm{~ms}$ & 16 \\
\hline OPG & $313.23 \mathrm{kPa}^{-1}$ & $0-4 \mathrm{kPa}$ & $28 \mathrm{~ms}$ & 17 \\
\hline
\end{tabular}




\begin{tabular}{lllll}
\hline Graphene & $17.2 \mathrm{kPa}^{-1}$ & $0-20 \mathrm{kPa}$ & - & 18 \\
\hline Graphene & $0.33 \mathrm{kPa}^{-1}$ & $0-5 \mathrm{kPa}$ & $<20 \mathrm{~ms}$ & 19 \\
\hline NICFs & $21710 \mathrm{kPa}-1$ & $50 \mathrm{~Pa}-100 \mathrm{kPa}$ & $30 \mathrm{~ms}$ & This work \\
\hline
\end{tabular}

Table S1. Comparison of the sensitivities within a linear pressure range between the oriented NICFs/PDMS pressure sensor and previous reported surface patterned microstructures.

\section{References}

(1)Gong, S.; Schwalb, W.; Wang, Y. W.; Chen, Y.; Tang, Y.; Si, J.; Shirinzadeh, B.; Cheng, W. L., A wearable and Highly Sensitive Pressure Sensor with Ultrathin Gold Nanowires. Nature Communications 2014, 5.

(2)Park, H.; Jeong, Y. R.; Yun, J.; Hong, S. Y.; Jin, S.; Lee, S. J.; Zi, G.; Ha, J. S., Stretchable Array of Highly Sensitive Pressure Sensors Consisting of Polyaniline Nanofibers and Au-Coated Polydimethylsiloxane Micropillars. Acs Nano 2015, 9 (10), 9974.

(3)Park, J.; Lee, Y.; Hong, J.; Ha, M.; Jung, Y. D.; Lim, H.; Kim, S. Y.; Ko, H., Giant Tunneling Piezoresistance of Composite Elastomers with Interlocked Microdome Arrays for Ultrasensitive and Multimodal Electronic Skins. Acs Nano 2014, 8 (5), 4689. (4)Bae, G. Y.; Pak, S. W.; Kim, D.; Lee, G.; Kim, D.; Chung, Y.; Cho, K., Linearly and Highly Pressure-Sensitive Electronic Skin Based on a Bioinspired Hierarchical Structural Array. Advanced Materials 2016, 28 (26), 5300.

(5)Xia, K. L.; Wang, C. Y.; Jian, M. Q.; Wang, Q.; Zhang, Y. Y., CVD Growth of Fingerprint-Like Patterned 3D Graphene Film for an Ultrasensitive Pressure Sensor. 
Nano Research 2018, 11 (2), 1124.

(6)Jian, M. Q.; Xia, K. L.; Wang, Q.; Yin, Z.; Wang, H. M.; Wang, C. Y.; Xie, H. H.; Zhang, M. C.; Zhang, Y. Y., Flexible and Highly Sensitive Pressure Sensors Based on Bionic Hierarchical Structures. Advanced Functional Materials 2017, 27 (9).

(7)Yao, H. B.; Ge, J.; Wang, C. F.; Wang, X.; Hu, W.; Zheng, Z. J.; Ni, Y.; Yu, S. H., A flexible and Highly Pressure-Sensitive Graphene-Polyurethane Sponge Based on Fractured Microstructure Design. Adv Mater 2013, 25 (46), 6692.

(8)Liu, M. M.; Pu, X.; Jiang, C. Y.; Liu, T.; Huang, X.; Chen, L. B.; Du, C. H.; Sun, J. M.; Hu, W. G.; Wang, Z. L., Large-Area All-Textile Pressure Sensors for Monitoring Human Motion and Physiological Signals. Advanced Materials 2017, 29 (41).

(9)Chen, Z.; Wang, Z.; Li, X.; Lin, Y.; Luo, N.; Long, M.; Zhao, N.; Xu, J. B., Flexible Piezoelectric-Induced Pressure Sensors for Static Measurements Based on Nanowires/Graphene Heterostructures. ACS Nano 2017, 11 (5), 4507.

(10)Lou, Z.; Chen, S.; Wang, L.; Jiang, K.; Shen, G., An Ultra-Sensitive and Rapid Response Speed Graphene Pressure Sensors for Electronic Skin and Health Monitoring. Nano Energy 2016, 23, 7.

(11)Wan, S.; Bi, H.; Zhou, Y.; Xie, X.; Su, S.; Yin, K.; Sun, L., Graphene Oxide as High-Performance Dielectric Materials for Capacitive Pressure Sensors. Carbon 2017, $114,209$.

(12)Tian, H.; Shu, Y.; Wang, X. F.; Mohammad, M. A.; Bie, Z.; Xie, Q. Y.; Li, C.; Mi, W. T.; Yang, Y.; Ren, T. L., A Graphene-Based Resistive Pressure Sensor with Record-High Sensitivity in a Wide Pressure Range. Sci Rep 2015, 5, 8603. 
(13)Jian, M.; Xia, K.; Wang, Q.; Yin, Z.; Wang, H.; Wang, C.; Xie, H.; Zhang, M.; Zhang, Y., Flexible and Highly Sensitive Pressure Sensors Based on Bionic Hierarchical Structures. Advanced Functional Materials 2017, 27 (9), 1606066.

(14)Chen, W.; Gui, X.; Liang, B.; Yang, R.; Zheng, Y.; Zhao, C.; Li, X.; Zhu, H.; Tang, Z., Structural Engineering for High Sensitivity, Ultrathin Pressure Sensors Based on Wrinkled Graphene and Anodic Aluminum Oxide Membrane. ACS Appl Mater Interfaces 2017, 9 (28), 24111.

(15)Ma, Y.; Yue, Y.; Zhang, H.; Cheng, F.; Zhao, W.; Rao, J.; Luo, S.; Wang, J.; Jiang, X.; Liu, Z.; Liu, N.; Gao, Y., 3D Synergistical MXene/Reduced Graphene Oxide Aerogel for a Piezoresistive Sensor. ACS Nano 2018, 12 (4), 3209.

(16)Yang, J.; Ye, Y.; Li, X.; Lü, X.; Chen, R., Flexible, Conductive, and Highly Pressure-Sensitive Graphene-Polyimide Foam for Pressure Sensor Application. Composites Science and Technology 2018, 164, 187.

(17)Deng, W.; Fang, Q.; Huang, H.; Zhou, X.; Ma, J.; Liu, Z., Oriented Arrangement: The Origin of Versatility for Porous Graphene Materials. Small 2017, 13 (34), 1701231. (18)Tao, L. Q.; Zhang, K. N.; Tian, H.; Liu, Y.; Wang, D. Y.; Chen, Y. Q.; Yang, Y.; Ren, T. L., Graphene-Paper Pressure Sensor for Detecting Human Motions. ACS Nano 2017, $11(9), 8790$.

(19)He, Z.; Chen, W.; Liang, B.; Liu, C.; Yang, L.; Lu, D.; Mo, Z.; Zhu, H.; Tang, Z.; Gui, X., Capacitive Pressure Sensor with High Sensitivity and Fast Response to Dynamic Interaction Based on Graphene and Porous Nylon Networks. ACS Appl Mater Interfaces 2018, 10 (15), 12816. 
